\title{
Helminth defence molecules-immunomodulators designed by parasites!
}

\author{
Mark W. Robinson ${ }^{1 *}$, Sheila Donnelly ${ }^{2}$ and John P. Dalton ${ }^{1,3}$ \\ ${ }^{1}$ Medical Biology Centre, School of Biological Sciences, Queen's University Belfast, Belfast, Northern Ireland \\ 2 The ithree Institute, University of Technology Sydney, Ultimo, Sydney, NSW, Australia \\ ${ }^{3}$ Institute of Parasitology, McGill University, St-Anne-de-Bellevue, QC, Canada \\ *Correspondence: mark.robinson@qub.ac.uk \\ Edited by: \\ Nádia S. Parachin, Universidade de Brasília-UnB, Brazil \\ Reviewed by: \\ Maxim Ryadnov, National Physical Laboratory, UK \\ Fernanda D. Silva, Universidade Federal do ABC, Brazil
}

Keywords: parasite, helminth, Fasciola, Schistosoma, trematodes, immunomodulator, helminth defence molecule, antimicrobial peptide

\section{HELMINTH DEFENCE MOLECULES (HDMs)—ANTIMICROBIALS OR IMMUNOMODULATORS?}

Parasitic helminths (worms) are one of the most successful animal groups in nature. They are large multicellular organisms and therefore cannot penetrate host cells but must reside inside tissue or organs. They infect over 1 billion people globally, mostly in tropical/sub-tropical regions, taking an enormous toll on animal and human health (Hotez et al., 2008). Although the main evolutionary driving force for parasitism may have been the ease of access to food this brought other challenges, most importantly the need to overcome expulsion by the immune responses of the host. Accordingly, helminths have evolved elaborate mechanisms to manage, suppress or manipulate the mammalian immune system.

It is generally thought that worms influence the host immune response by secreting factors into their environment-the host parasite interface. Over the past 10 years the application of proteomics techniques has allowed us to identify molecules secreted by helminths. Although the exact complement of secretory molecules differs between species, most helminths release proteolytic enzymes with endoand exo-peptidase activities (Robinson et al., 2008a,b), antioxidants such as glutathione- $S$ transferase and peroxiredoxin (Jefferies et al., 2001; Donnelly et al., 2008; Robinson et al., 2009) and other molecules with a range of biochemical activities including protease inhibitors and metabolic enzymes. Whilst the biological function of a few of these helminth molecules are well defined, this is generally restricted to those that possess evolutionarily conserved catalytic domains/active site residues that are easily identified using bioinformatics search tools (e.g., Fasciola cysteine proteases function in fluke nutrition; Lowther et al., 2009). However, many helminths also secrete a range of molecules whose primary sequences offer no clue as to their biochemical activity or biological function.

During our on-going analysis of the secretory proteome (i.e., the secretome) of the helminth Fasciola hepatica, a parasite that infects its host via the intestine following ingestion and then migrates to the liver, we identified a novel and abundant $8 \mathrm{kDa}$ protein. The protein contained a secretory signal peptide but BLAST analysis of its primary sequence failed to infer a function. However, structural studies revealed that a 37 amino acid C-terminal region adopted a similar secondary structure (amphipathic $\alpha$-helix) to a number of peptides with known antimicrobial and/or immunomodulatory functions, most particularly mammalian LL-37 (Robinson et al., 2011). Phylogenetic analysis discovered that these secretory proteins are conserved across the major trematode species that collectively infect $>1$ billion humans, including the liver flukes Clonorchis sinensis, Paragonimus westermani, and Opisthorchis viverrini and the blood flukes Schistosoma mansoni and S. japonicum (Robinson et al., 2011). Accordingly, by analogy with the mammalian antimicrobial and/or immunomodulators we hypothesized that these helminth molecules may play a critical role in the parasites interaction with its host and thus named them helminth defence molecules (HDMs). This hypothesis, however, posed the question that is central to understanding how they may perform this function, namely, are HDMs antimicrobial or immunomodulatory peptides, or both?

\section{HDMS DO NOT POSSESS}

\section{ANTIMICROBIAL OR HAEMOLYTIC} ACTIVITY

To begin to address this question, we compared the ability of helminthderived HDMs with several well-known mammalian host-derived antimicrobial peptides [AMPs; also termed host defence peptides (HDPs) where they display immunomodulatory activities] to directly kill clinically-relevant microorganisms (Thivierge et al., 2013). The antimicrobial effect of AMPs/HDPs is attributed to the ability of their amphipathic helices to bind, and thus disrupt, negatively charged bacterial cell membranes (Hancock and Chapple, 1999). Despite having similar biochemical/biophysical properties to the host-derived AMPs/HDPs including LL-37, CRAMP, SMAP-29, and BMAP-28 which variously showed activity against both gram-negative (Escherichia coli, Pseudomonas aeruginosa, and Salmonella typhimurium) and gram-positive (Staphylococcus epidermis and Staphylococcus aureus) bacteria, none of the HDMs tested demonstrated 
bactericidal activity against any species of bacteria at the concentrations tested (<0.25-128 $\mu \mathrm{g} / \mathrm{ml}$; Thivierge et al., 2013).

Previous studies had also shown that AMPs/HDPs are cytotoxic to kinetoplastid protozoan parasites such as Leishmania major (Lynn et al., 2011), Trypanosoma cruzi (Haines et al., 2009), and the apicomplexan protozoan parasites Cryptosporidium sp. (Carryn et al., 2012). In our studies, whilst we confirmed that several vertebrate AMPs/HDPs killed the apicomplexan protozoan parasite Cryptosporidium parvum and C. hominis in vitro at concentrations as low as $0.025 \mu \mathrm{M}$ none of the HDMs tested displayed any parasiticidal activity even at $2.5 \mu \mathrm{M}$ (Thivierge et al., 2013).

The predominant mechanism of AMP/HDP bactericidal activity is the formation of pores in the membrane lipid bilayer, destroying its integrity and causing cell death (Oren and Shai, 1998). However, this effect is not specific to bacterial cells since eukaryotic cells can also be lysed via this mechanism (Ciornei et al., 2005). Whilst the mammalian AMPs/HDPs rapidly lysed red blood cells in a concentrationdependent manner (from 8 to $256 \mu \mathrm{g} / \mathrm{ml}$ ), the HDMs did not induce significant lysis at equivalent concentrations (Thivierge et al., 2013). Using a fluorescent membrane-impermeant dye we demonstrated that the mammalian peptides LL-37, CRAMP, SMAP-29, and BMAP-28 $(50 \mu \mathrm{M})$ induced the formation of pores in a murine macrophage cell line and in general, were cytotoxic at concentrations of $>25 \mu \mathrm{M}$. However, at the same concentrations none of the helminth peptides exhibited these lytic or cytotoxic effects (Thivierge et al., 2013).

The contrasting effects of the mammalian AMPs/HDPs and the helminth HDMs may be due to the targeting of specific membrane components by HDMs necessary for internalization into host cells rather than non-specific "carpet" binding to the phospholipid bilayer that may occur with the AMPs/HDPs (Brender et al., 2012). We have shown that F. hepatica HDM (FhHDM-1) binds to host macrophage plasma membrane lipid rafts, possibly via selective interaction with cholesterol, before being internalized by endocytosis (Robinson et al.,
2012). In contrast, human LL-37 cannot bind to cholesterol; indeed its presence strongly reduces the ability of LL-37 to interact with phospholipid membranes (Sood and Kinnunen, 2008). Whilst the precise mechanism(s) governing the selectivity of the HDMs vs. AMPs/HDPs is not fully understood, our observations show that, rather than simply destroying host cells by lysis, HDMs have evolved specifically to interact with host cell membranes without causing their disruption.

\section{HDMs DISPLAY A VARIETY OF IMMUNOMODULATORY ACTIVITIES}

Parasitic helminths secrete a range of soluble effector molecules that modulate host immune responses in a myriad of ways to establish an environment that facilitates their survival and a prolonged reproductive phase (reviewed by Harnett and Harnett, 2010). We have previously shown that $F$. hepatica and S. mansoni secrete molecules with specific immunomodulatory functions: peroxiredoxin (Prx) promotes the development of host Th2 responses via the induction of M2 macrophages (Donnelly et al., 2005, 2008) and cathepsin L1 (FhCL1) inhibits the macrophage MyD88-independant, TRIF-dependant signaling pathway via cleavage of toll-like receptor (TLR) 3 within the endosome (Donnelly et al., 2010). However, our recent studies have shown that HDMs are "utility players" in the host-parasite interaction and exert multiple effects on host immune cells.

In order to infect their mammalian host, the infective stage of $F$. hepatica, termed newly excysted juveniles (NEJs), secrete an array of cysteine peptidases including cathepsins $\mathrm{B}$ and cathepsins $\mathrm{L}$ that digest a path through the intestinal wall (McGonigle et al., 2008; Robinson et al., 2009). Despite this loss of barrier function of the intestinal epithelium and consequent translocation of luminal antigens (bacteria and their toxins) into the circulation, potent host responses such as septicaemia are not common events during helminth infections. We found that FhHDM-1 binds directly to E. coli lipopolysaccharide (LPS) preventing its interaction with the TLR4/MD2/CD14 complex on the macrophage surface (Robinson et al., 2011). FhHDM1 also exhibited a striking ability to protect mice against LPS-induced inflammation by preventing the release of inflammatory mediators (TNF and IL$1 \beta$ ) from macrophages (Robinson et al., 2011).

IFN $\gamma$ is one of the key cytokines in the innate immune response to intracellular pathogens, and augments cellular responses to TLR ligands such as bacterial LPS (Held et al., 1999; Schroder et al., 2006). We also found that, like the mammalian peptides LL-37, CRAMP, SMAP-29, and BMAP-28, HDMs significantly inhibited macrophage TNF production in response to combined stimulation with LPS and IFN $\gamma$ (Thivierge et al., 2013). Both AMPs/HDPs and HDMs can seemingly also inhibit inflammatory macrophages using mechanisms that are independent of direct binding to LPS (Brown et al., 2011; Thivierge et al., 2013). Thus, the secretion of HDMs by the parasite may protect the host against excessive bacterial-induced inflammation that would otherwise occur during migration of the parasite through the host intestinal wall due to concurrent translocation of luminal bacteria. By offering this protection the parasite enhances the survival of its host and, accordingly, its own longevity.

Confocal microscopy, using fluorescently labeled peptides, has shown that after initial interaction with lipid rafts on the macrophage surface, FhHDM-1 enters the cell via the endolysosomal pathway (Robinson et al., 2012). FhHDM-1 is cleaved by endogenous (host) cathepsin L to specifically release a C-terminal peptide (containing the conserved HDM amphipathic helix) which then prevents the acidification of the endolysosomal compartments by inhibiting vacuolar (v) ATPase activity. Uncoupling endolysosomal acidification impedes macrophage antigen processing by proteases, such as cathepsin L, preventing the presentation of peptides at the cell surface in conjunction with MHC class II to CD4 + T cells (Robinson et al., 2012). By suppressing the antigen presenting function of host macrophages, HDM indirectly impairs the subsequent development of adaptive immune responses 
against the parasite. However, by altering the secretion of immunoglobulins from activated B cells, HDMs are also capable of directly influencing the host adaptive response. HDMs enhanced the IL-4 induced production of IgG1 and suppressed the release of IgG2a from murine $\mathrm{B}$ cells in response to IFN $\gamma$ (Thivierge et al., 2013). This is consistent with a wound healing scenario (Nishio et al., 2009) which, again, may serve to protect the host from helminth-induced tissue damage.

\section{PARASITE-DESIGNED HDMs MAY HAVE THERAPEUTIC POTENTIAL}

The immune-modulatory properties of mammalian HDPs, and in particular their ability to prevent excessive immunopathology associated with bacterial sepsis, has attracted interest in exploiting these as anti-infectives and immunotherapeutic agents (Easton et al., 2009). However, their clinical development has been hampered by the occurrence of toxic off-target effects and cell lysis. Efforts to improve delivery of HDPs to their desired site of action (e.g., by conjugation with targeting moieties) are on-going with the aim of enhancing efficacy whilst reducing deleterious side effects (Devocelle, 2012). However, helminth-derived HDMs may represent a more attractive therapeutic option: they show all the potent immunomodulatory effects of the HDPs without the cytotoxic and cytolytic effects (Thivierge et al., 2013). We are currently determining the translatability of the immune-modulatory effect of HDMs from murine to human cells rather than screening an array of animal models of disease (Robinson et al., 2013) and we are hopeful that the therapeutic potential of these parasite-designed molecules can be realised (Donnelly et al., 2011).

\section{REFERENCES}

Brender, J. R., McHenry, A. J., and Ramamoorthy, A. (2012). Does cholesterol play a role in the bacterial selectivity of antimicrobial peptides? Front. Immunol. 3:195. doi: 10.3389/fimmu.2012.00195

Brown, K. L., Poon, G. F., Birkenhead, D., Pena, O. M., Falsafi, R., Dahlgren, C., et al. (2011). Host defense peptide LL-37 selectively reduces proinflammatory macrophage responses. J. Immunol. 186, 5497-5505. doi: 10.4049/jimmunol.1002508
Carryn, S., Schaefer, D. A., Imboden, M., Homan, E. J., Bremel, R. D., and Riggs, M. W. (2012). Phospholipases and cationic peptides inhibit Cryptosporidium parvum sporozoiteinfectivity by parasiticidal and non-parasiticidal mechanisms. J. Parasitol. 98, 199-204. doi: 10.1645/GE-2822.1

Ciornei, C. D., Sigurdardottir, T., Schmidtchen, A., and Bodelsson, M. (2005). Antimicrobial and chemoattractant activity, lipopolysaccharide neutralization, cytotoxicity, and inhibition by serum of analogs of human cathelicidin LL-37. Antimicrob. Agents Chemother. 49, 2845-2850. doi: 10.1128/AAC.49.7.28452850.2005

Devocelle, M. (2012). Targeted antimicrobial peptides. Front. Immunol. 3:309. doi: 10.3389/fimmu.2012.00309

Donnelly, S., O’Neill, S. M., Sekiya, M., Mulcahy, G., and Dalton, J. P. (2005). Thioredoxin peroxidase secreted by Fasciola hepatica induces the alternative activation of macrophages. Infect. Immun. 73, 166-173. doi: 10.1128/IAI.73.1.166-173.2005

Donnelly, S., O’Neill, S. M., Stack, C. M., Robinson, M. W., Turnbull, L., Whitchurch, C., et al. (2010). Helminth cysteine proteases inhibit TRIFdependent activation of macrophages via degradation of TLR3. J. Biol. Chem. 285, 3383-3392. doi: 10.1074/jbc.m109.060368

Donnelly, S., Robinson, M. W., Dalton, J. P., and To, J. (2011). Immune modulating agents and uses therefor. PCT/AU2011/001402.

Donnelly, S., Stack, C. M., O’Neill, S. M., Sayed, A. A., Williams, D. L., and Dalton, J. P. (2008). Helminth 2-Cys peroxiredoxin drives Th2 responses through a mechanism involving alternatively activated macrophages. FASEB J. 22, 4022-4032. doi: 10.1096/fj.08-106278

Easton, D. M., Nijnik, A., Mayer, M. L., and Hancock, R. E. (2009). Potential of immunomodulatory host defense peptides as novel antiinfectives. Trends Biotechnol. 27, 582-590. doi: 10.1016/j.tibtech.2009.07.004

Haines, L. R., Thomas, J. M., Jackson, A. M., Eyford, B. A., Razavi, M., Watson, C. N., et al. (2009). Killing of trypanosomatid parasites by a modified bovine host defense peptide, BMAP-18. PLoS Negl. Trop. Dis. 3:e373. doi: 10.1371/journal.pntd.0000373

Hancock, R. E., and Chapple, D. S. (1999). Peptide antibiotics. Antimicrob. Agents Chemother. 43, 1317-1323.

Harnett, W., and Harnett, M. M. (2010). Helminthderived immunomodulators: can understanding the worm produce the pill? Nat. Rev. Immunol. 10, 278-284. doi: 10.1038/nri2730

Held, T. K., Weihua, X., Yuan, L., Kalvakolanu, D. V., and Cross, A. S. (1999). Gamma interferon augments macrophage activation by lipopolysaccharide by two distinct mechanisms, at the signal transduction level and via an autocrine mechanism involving tumor necrosis factor alpha and interleukin-1. Infect. Immun. 67, 206-212.

Hotez, P. J., Brindley, P. J., Bethony, J. M., King, C. H., Pearce, E. J., and Jacobson, J. (2008). Helminth infections: the great neglected tropical diseases. J. Clin. Invest. 118, 1311-1321. doi: 10.1172/JCI34261

Jefferies, J. R., Campbell, A. M., van Rossum, A. J., Barrett, J., and Brophy, P. M. (2001). Proteomic analysis of Fasciola hepatica excretory-secretory products. Proteomics 1, 1128-1132.

Lowther, J., Robinson, M. W., Donnelly, S. M., Xu, W., Stack, C. M., Matthews, J. M., et al. (2009). The importance of $\mathrm{pH}$ in regulating the function of Fasciola hepatica cathepsin L1 cysteine protease. PLoS Negl. Trop. Dis. 3:e369. doi: 10.1371/journal.pntd.0000369

Lynn, M. A., Kindrachuk, J., Marr, A. K., Jenssen, H., Pante, N., Elliott, M. R., et al. (2011). Effect of BMAP-28 antimicrobial peptides on Leishmania major promastigote and amastigote growth: role of leishmanolysin in parasite survival. PLoS Negl. Trop. Dis. 5:e1141. doi: 10.1371/journal.pntd.0001141

McGonigle, L., Mousley, A., Marks, N. J., Brennan, G. P., Dalton, J. P., Spithill, T. W., et al. (2008). The silencing of cysteine proteases in Fasciola hepatica newly excysted juveniles using RNA interference reduces gut penetration. Int. J. Parasitol. 38, 149-155. doi: 10.1016/j.ijpara.2007. 10.007

Nishio, N., Ito, S., Suzuki, H., and Isobe, K. (2009). Antibodies to wounded tissue enhance cutaneous wound healing. Immunology 128, 369-380. doi: 10.1111/j.1365-2567.2009.03119.x

Oren, Z., and Shai, Y. (1998). Mode of action of linear amphipathic alpha-helical antimicrobial peptides. Biopolymers 47, 451-463.

Robinson, M. W., Alvarado, R., To, J., Hutchinson, A. T., Dowdell, S. N., Lund, M., et al. (2012). A helminth cathelicidin-like protein suppresses antigen processing and presentation in macrophages via inhibition of lysosomal vATPase. FASEB J. 26, 4614-4627. doi: 10.1096/fj.12213876

Robinson, M. W., Dalton, J. P., and Donnelly, S. (2008a). Helminth pathogen cathepsin proteases: it's a family affair. Trends Biochem. Sci. 33, 601-608. doi: 10.1016/j.tibs.2008.09.001

Robinson, M. W., Tort, J. F., Wong, E., Donnelly, S. M., Lowther, J., Xu, W., et al. (2008b). Proteomics and phylogenetic analysis of the cathepsin L protease family of the helminth pathogen, Fasciola hepatica: expansion of a repertoire of virulence-associated factors. Mol. Cell. Proteomics 7, 1111-1123. doi: 10.1074/mcp.M700560MCP200

Robinson, M. W., Dalton, J. P., O'Brien, B. A., and Donnelly, S. (2013). Fasciola hepatica: the therapeutic potential of a worm secretome. Int. J. Parasitol. 43, 283-291.

Robinson, M. W., Donnelly, S., Hutchinson, A. T., To, J., Taylor, N. L., Norton, R. S., et al. (2011). A family of helminth molecules that modulate innate cell responses via molecular mimicry of host antimicrobial peptides. PLoS Pathog. 7:e1002042. doi: 10.1371/journal.ppat.1002042

Robinson, M. W., Menon, R., Donnelly, S. M., Dalton, J. P., and Ranganathan, S. (2009). An integrated transcriptomic and proteomic analysis of the secretome of the helminth pathogen, Fasciola hepatica: proteins associated with invasion and infection of the mammalian host. Mol. Cell. Proteomics 8, 1891-1907. doi: 10.1074/mcp.M900045-MCP200

Schroder, K., Sweet, M. J., and Hume, D. A. (2006). Signal integration between IFNgamma and TLR signalling pathways in 
macrophages. Immunobiology 211, 511-524. doi: 10.1016/j.imbio.2006.05.007

Sood, R., and Kinnunen, P. K. (2008). Cholesterol, lanosterol, and ergosterol attenuate the membrane association of LL-37(W27F) and temporin L. Biochim. Biophys. Acta 1778, 1460-1466. doi: 10.1016/j.bbamem.2008. 02.014

Thivierge, K., Cotton, S., Schaefer, D. A., Riggs, M. W., To, J., Lund, M. E., et al. (2013). Cathelicidin-like helminth defence molecules (HDMs): absence of cytotoxic, anti-microbial and anti-protozoan activities imply a specific adaptation to immune modulation. PLoS Negl. Trop. Dis. 7:e2307. doi: 10.1371/journal.pntd. 0002307

Received: 22 July 2013; accepted: 14 September 2013; published online: 01 October 2013.

Citation: Robinson MW, Donnelly $S$ and Dalton JP (2013) Helminth defence moleculesimmunomodulators designed by parasites! Front. Microbiol. 4:296. doi: 10.3389/fmicb.2013.00296
This article was submitted to Antimicrobials, Resistance and Chemotherapy, a section of the journal Frontiers in Microbiology.

Copyright (c) 2013 Robinson, Donnelly and Dalton. This is an open-access article distributed under the terms of the Creative Commons Attribution License (CC BY). The use, distribution or reproduction in other forums is permitted, provided the original author(s) or licensor are credited and that the original publication in this journal is cited, in accordance with accepted academic practice. No use, distribution or reproduction is permitted which does not comply with these terms. 